\title{
A Novel Self-Regulating and Intelligence Meta-Heuristic-Fuzzy Approach for Integrated and Optimal Human Resource Allocation in Normal and Critical Conditions
}

\author{
Hamidreza Seifi $^{1}\left(\mathbb{D}^{\cdot}\right.$ Naser Shams $^{1} \cdot$ Kaveh Mohammad Cyrus $^{1}$
}

Received: 24 February 2021/Revised: 20 May 2021/Accepted: 25 May 2021/Published online: 20 June 2021

(C) Taiwan Fuzzy Systems Association 2021

\begin{abstract}
This study aims to present a novel Self-regulating and Intelligence Meta-Heuristic-Fuzzy approach (As Methodological Contribution) for integrated and optimal Human Resource Allocation (HRA) in normal and critical conditions at SMEs (As Conceptual Contribution). In this research, a mathematical model of human resource allocation problem is presented, and then Sugeno Fuzzy Inference (SFI) model is used in the tasks rate adjustment layer. The SFI model is the main part of developing Gray Wolf Optimization (GWO) algorithm to reach the integrated and optimal allocation of available human resources under self-regulating attribute in the novel approach. The novel approach has tested and compared to the best researches using data previous researches and by the top five proposed methods in the researches (Includes: SGA, PRS, SRS, MIP, HM) based on three methods of evaluating the quality of solutions (GA-FSGS, MP-FSGS, GA-SGS). The results showed that increase of $\Omega$ from 15,000 to 25,000, and HM and SGA clearly performed better than other previous cases in the larger B100 and B200 datasets. Also, it is verified that the method had better results compare to all previous solving methods, and the quality of the solutions have been the best.
\end{abstract}

Hamidreza Seifi

hamid.baf@gmail.com

Naser Shams

nshams@aut.ac.ir

Kaveh Mohammad Cyrus

cyrusk@aut.ac.ir

1 Department of Industrial Engineering and Systems Management, Amirkabir University of Technology, Tehran, Iran
Keywords Human resource allocation (HRA) · Normal and critical conditions - Self-regulating and intelligence approach (SRI) - Grey wolf optimization algorithm $(\mathrm{GWO}) \cdot$ Sugeno fuzzy interference model (SFI)

\section{Introduction}

Today, effective human resource management plays an important role in the success of organizations and organizations try to allocate the best people for organizational activities in order to achieve high efficiency of limited resources [1]. However, make decisions for human capital allocation into different units of the organization and find the efficient combination of HR in the organization are difficulties of human resource management [2]. But human resources are the most valuable resource of today's organizations and this source forms organizational decisions, solves organization problems and objectify productivity. However, increasing the productivity of organizations and their increasing development requires the growth and productivity of employees [3]. More importantly, the importance and position of human resources and efficient allocation of human resources in organization have led to comprehensive approaches for HRA problems, especially in critical conditions [4]. But what is HRA problem? HRA problem is a resource-constrained planning problem that tries assuming that human resources are renewable but difficult to provide this resource immediately, set a certain number of trained and appropriate human resources in different steps of the process till reaches highest level of individual and organizational performance with the lowest cost, the shortest possible time and the highest appropriate quality [5]. This problem includes activities that must be planned according to resource constraints and the priorities 
in such a way till the defined goals are reached for one or more ongoing projects [6]. From the point of view of project planning, the human resource allocation problem can be considered a resource-constrained planning problem. However, project planning is an important activity in project management [7].

This type of problem has become a standard problem for project planning in the literature in recent decades. Albeit, the Human resources are renewable sources because their full capacity is available in each period. We have $k$ renewable sources that is denoted by $k=1, K$. For each resource $k$, the availability is assumed to be constant over time and denoted by $\mathbf{R}_{\mathrm{k}}$. Activity $j$ requires $\mathrm{r}_{\mathrm{jk}}$ units from source $k$ in each ongoing period. We consider two activities $j=0$ and $j=J+1$ to indicate the project's beginning and end, respectively. Both of these activities are virtual activities with a duration time of 0 and no need for resources. Also, it is assumed that all information is crisp and already defined. The parameters are assumed to be non-negative and integers. One resource allocation is the allocation of $S_{\mathrm{j}}$ start times for activities $j=0,1, J+1$ $[8,9]$. For the primary resource allocation problem, the goal is to find a program that leads to the soonest time of project ending. In other words, the minimum total time to complete the project. Vanhoucke [10] showed that resource-constrained planning problem is an NP-Hard problem.

But why should the researchers address the issue of human resource allocation in this study? This research has been done because of three important reasons:

1. The issue of human resource allocation has always been a growing issue and it has not yet been fully resolved through various proposed methods and solutions. This claim verifies according to the information in Table 1.

2. The evaluation results of previous research show that attention to the issue of human resource allocation is an separated and island view and this issue has not been resolved integrated and comprehensively.

3. However, HR of any organization should always be in proper condition in both non-critical and critical situations, and they don't make challenge for organization, but there is no a solution to solve the related problems in critical situations (absence or leaving employees due to forced or unforeseen events or pervasive events such as COVID-19, etc.) until the beginning of 2021 .

Albeit, Trishtler et al. [9] tried to find a solution to this important issue. His research was only able to provide the necessary control before and after the crisis, and he was hoped that an intelligent solution can provide to solve the problem in critical and non-critical situations with minimal human intervention (due to computational error). So, this is the research gap, and, in this research an attempt has been carried to integrate the GWO algorithm, the Sugeno fuzzy inference method-as a new algorithm that is expected to be able to self-regulate the parameters and to provide the optimal solution/solutions for the first time even in case of changing the objectives of the problem.

\section{Literature Review}

The HRA has been considered scientifically and comprehensively for more than 3 decades. So, there has been a lot of research on the subject in scientific community that most of those have been in staff allocation to the tasks or organizational positions. Table 1 shows lists of the internal and external researches related to the HRA problem.

According to the research presented in Table 1 (The models and methods used in these researches), there are two weak in these researches. First, the linear programming models cannot control the multi-objective issues of HRA, whereas these problems are particularly include many uncertainties (they have fuzzy context) in their natures. Second, according to the dynamic nature of HRA objectives, the mathematics models should cover the evolving structures in organizations and the uncertainties in organizations' environment. While, this is obvious that all previous researches have tried to achieve a specific pattern of time, cost, or quality through fixation the problem environment and setting boundaries for the parameters and the objectives. But, the environment and parameters of the HRA problem are changeable and flexible, and therefore this study has tried to assume that the parameters affecting the issue of HRA are variable. Then tries to do a simulation of the HRA in normal (before and after the crisis) and under critical conditions (during occurrence of crisis). On the other hand, various companies and organizations suffer from disruption in the work process under normal and acute crisis conditions (Because of the absence of one or more employees or leaving the job by $\mathrm{HR}$ or firing employees).

Also, in an organization as an integrated system, tasks and people are interdependent, and they are dependent on each other. It means that we need an integrated and intelligence method to do HRA in organizations, continuously. Even, if it is possible, we need a self-regulating and intelligence method that can optimize its structure according to the changes in the objectives and achieve optimal solutions. For this valuable it is issued a novel combination of GWO algorithm as a member of local optimization algorithms and Sugeno Fuzzy Interference Model to the algorithm's body to reach the self-regulating 
Table 1 A review on literature of HRA problem

\begin{tabular}{|c|c|c|c|c|}
\hline \multirow[t]{2}{*}{ Author/s (Year) } & \multirow[t]{2}{*}{ Allocation based on: } & \multicolumn{2}{|c|}{ Conditions } & \multirow[t]{2}{*}{ Methods/Approaches } \\
\hline & & Nor & Criti & \\
\hline Kwak et al. [11] & Activities & & * & Fuzzy Approach \\
\hline L.Saaty et al. [12] & Activities & & $*$ & AHP Method \\
\hline $\begin{array}{l}\text { Alvarez-Valdés } \\
\text { et al. [8] }\end{array}$ & Renewable activity & & * & GRASP Algorithm \\
\hline Lin and Gen [13] & Multi-functional & & $*$ & MOGA \\
\hline Estellon et al. [14] & Task Scheduling & & $*$ & A Local Searching Algorithm \\
\hline Filho et al. [15] & Activities & & $*$ & Constraint Satisfaction approach \\
\hline Perez et al. [16] & Multi-functional & & $*$ & Sociometric Techniques \\
\hline $\begin{array}{l}\text { Cabanillas et al. } \\
\text { [17] }\end{array}$ & Business Process & & $*$ & Semantic Web Approach \\
\hline Chien et al. [18] & Multi-functional & & $*$ & Agent-based Negotiation Mechanism \\
\hline $\begin{array}{l}\text { Stylianou and } \\
\text { Andreou [19] }\end{array}$ & Project Scheduling & & * & Software Project Management Method \\
\hline $\begin{array}{l}\text { Ponsteen and } \\
\text { Kusters [20] }\end{array}$ & Automated Resource & & * & Center Heuristic Algorithm \\
\hline Park et al. [21] & Experience & & $*$ & GA \\
\hline $\begin{array}{l}\text { Wibisono et al. } \\
\text { [22] }\end{array}$ & Performance & & * & Naïve Bayes \\
\hline Roque et al. [23] & Task Similarities & & $*$ & GA and Random Searching Approach \\
\hline Lilia[24] & Competency & & * & Inverse Optimization Method \\
\hline $\begin{array}{l}\text { Bouajaja and Dridi } \\
\text { [25] }\end{array}$ & Review Paper & & * & - \\
\hline Aviso et al. [26] & Climate Critics & & * & P-Graph Approach \\
\hline Tritschler et al. [9] & Resource Flexibility & & $*$ & Mathematical Modeling, GA \\
\hline Yua et al. [5] & Human Suffering & & $*$ & Dynamic Programing \\
\hline Arias et al. [2] & Review Paper & & $*$ & - \\
\hline Arias et al. [4] & Activities & & * & A Systematic Mapping, BPM \\
\hline Arias et al. [27] & Activities Efficiency & & $*$ & On-Demand and Batch Scenarios \\
\hline Ersmus et al. [3] & HR Abilities & & * & Flichman Taxonomy Approach \\
\hline $\begin{array}{l}\text { Yousefi and } \\
\text { Yousefi [7] }\end{array}$ & Time minimization & & * & ANFIS, FNN, RNN, ICA \\
\hline $\begin{array}{l}\text { Ballesteros-Pérez } \\
\text { et al. [28] }\end{array}$ & Expert & & * & GA \\
\hline Dabrian et al. [1] & Expert & & * & Dynamic Modeling \\
\hline Xiao [28] & Expert & & * & Fuzzy PSO Algorithm \\
\hline $\begin{array}{l}\text { Khanizad and } \\
\text { Montazere [29] }\end{array}$ & Operational Performance & & & \\
\hline Current Study & $\begin{array}{l}\text { Experts, Activities, Minimization of the times and the } \\
\text { costs, Maximization of the quality }\end{array}$ & $*$ & $*$ & $\begin{array}{l}\text { Mathematical Modeling, Sugeno Fuzzy } \\
\text { Inteeference Model, GWO Algorithm }\end{array}$ \\
\hline
\end{tabular}

attribute. It will convert the simple algorithm to an integrated and intelligent algorithm, and solve the HRA. This solution can be a new success in the major of HRA and optimal planning to control the undesirable impacts of various critical conditions on HR performance and organizational performance. The critical conditions can be employee absence, pervasive diseases such as Covid-19 or Influenzas, the occurrence of unexpected events that lead the organization to critical situations, etc. 


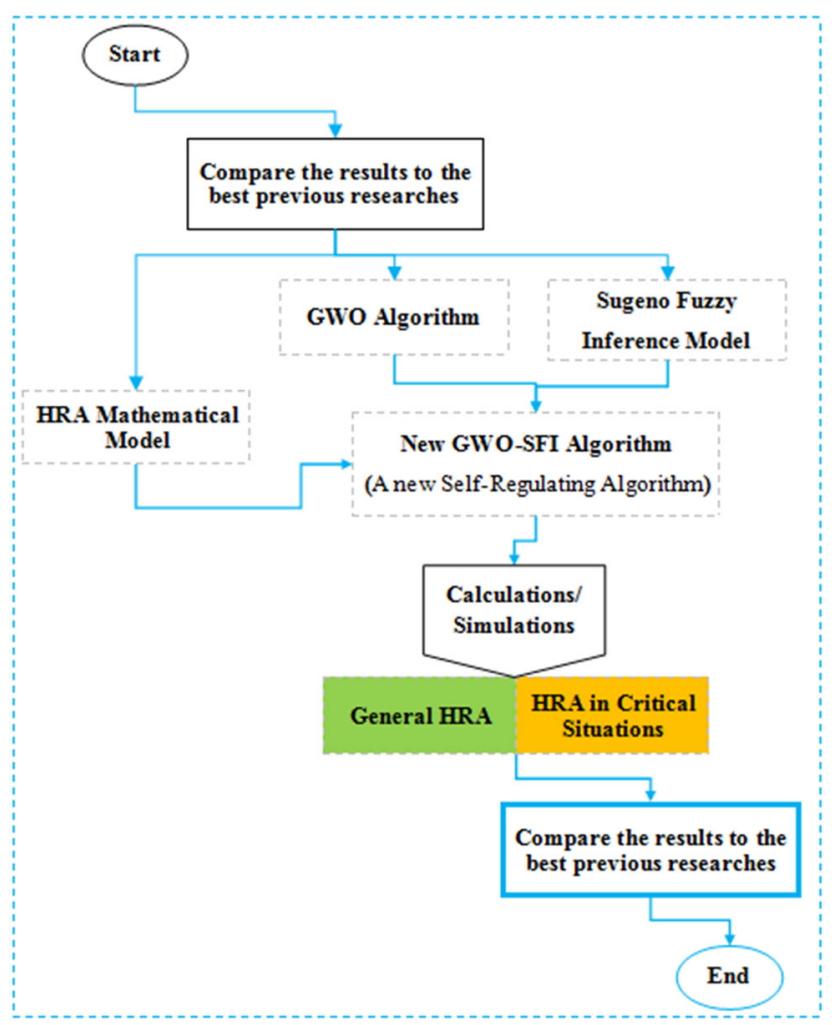

Fig. 1 Flowchart of the research

\subsection{Methodology}

This research try to do a meticulous allocation using a novel method. The allocation operation will do based on flowchart that illustrated in Fig. 1. According to Fig. 1, the proposed method is a novel approach that the authors will propose it be able to cover the research gap in the HRA problem (i.e. according to the intent flexibility and the variability of organizations environment, it suggested that there is a new method that this method is able and efficiency to do the allocation in critical situation or in general).

\subsection{HRA Problem Formulation}

In this section, the formulation of the HRA problem and its explanations are given using Alvarez-Valdés et al. [8] and Tritschler et al. [9].

\subsection{Improvements in GWO Algorithm}

In this research, developed GWO algorithm is used to solve the problem. This algorithm is based on the social lifestyle and hunting behavior of gray wolves. In this algorithm, in order to model the social behavior of wolves, a random population of solutions is generated and the first optimal solution is called alpha; the second and third optimal solutions are introduced as beta and delta, respectively, Also, other solutions are considered as a category of omega wolves. The GWO algorithm uses three solutions: alpha, $\beta$, and $\delta$ to guide the hunt (optimization solution), and the $\omega$ solution follow these three. In order to model three phases, it is necessary to first determine the points around the prey, then move towards the prey, and finally attack the prey. In the algorithm, the search process is created by creating a random population of gray wolves (candidate solutions). During the repetition, alpha, beta, and delta wolves estimate the probable position of prey. All available solutions then update their distance from the prey. The parameter is reduced from 2 to 0 to emphasize local and global search. Candidate solutions when $|A|>1$ tend to move away from the bait and when $|A|<1$ tends to bait. Local and global search is highly dependent on how parameter A works and how elitism is chosen to update the population of candidate solutions. This approach will eventually bring $\alpha, \beta$, and $\delta$ values closer together, reducing local and global searches for global optimization. Reducing local and global searches will predispose the GWO algorithm to fall into the optimal local trap. 
The standard gray wolf algorithm, when $|\mathrm{A}|<1$, uses three optimal values $\alpha, \beta$ and $\delta$ to search locally (attack to the prey). In order to improve the performance of gray wolves when attacking prey, several $\delta$-class wolves are added to the $\alpha$-wolf category. In fact, by increasing the effect of $\alpha$-solutions and decreasing the effect of $\delta$-solutions, it works to improve random neighborhood search. Equations (1) to (3) show how the improved gray wolf algorithm works to improve local search performance:

$\overrightarrow{D_{j}}=\left|\overrightarrow{C_{i}} \cdot \overrightarrow{X_{j}}-\vec{X}\right|$

$\overrightarrow{X_{i}}=\overrightarrow{X_{j}}-\overrightarrow{A_{1}}\left(\overrightarrow{D_{j}}\right)$

$(i, j) \in\{(1, \alpha),(2, \alpha),(3, \beta),(4,6)\}$

After calculating amounts of $X_{i}$, the $\mathrm{X}$ point is updated as follows:

$\vec{X}(t+1)=\frac{\overline{X_{1+} X_{2}}+\overline{\left(X_{3}-\overline{X_{4}}\right)}}{3}$

When $|A|>1$, the GWO algorithm searches the entire search space using the three optimal values $\alpha, \beta$ and $\delta$. The final position will be at a random location in a circle defined by the positions $\alpha, \beta$ and $\delta$ in the searching space. After repeating the algorithm, optimal solutions include $\alpha$, $\beta$ and $\delta$ converge, and thus the search space will reduce to find new points.

In this study, three optimal solutions from the current response population are used to improve the global search of the standard GWO algorithm. These include the best optimal solution of population $\alpha^{\prime}$, the second and third optimal solutions are $\beta$ ' and $\delta$,', respectively. Using these three values, we have some expands in search space in the algorithm due to the change in their value each time the algorithm is executed. Equations (5) to (8) show how the improved algorithm works during a global search:

$\overrightarrow{D_{j}}=\left|\overrightarrow{C_{i}} \cdot \overrightarrow{X_{j}}-\vec{X}\right|$

$\overrightarrow{X_{i}}=\overrightarrow{X_{j}}-\overrightarrow{A_{1}}\left(\overrightarrow{D_{j}}\right)$

$(i, j) \in\left\{\left(1, \alpha^{\prime}\right),\left(2, \beta^{\prime}\right),\left(3, \delta^{\prime}\right)\right\}$

After calculating amounts of $X_{i}$, the $\mathrm{X}$ point is updated as follows:

$\vec{X}(t+1)=\frac{\overrightarrow{x_{1}}+\overrightarrow{x_{2}}+\overrightarrow{x_{3}}}{3}$

Now, we describe how to set the transmission rate, and application a fuzzy controller to optimize the algorithm in this research, too.

\subsubsection{Set Task Transmission Rate}

In this section, regulation of data transmission rate is stated. First, the problem of task transmission rates is described through task divider nodes. The proposed method to solve this problem is presented in the final section. The method is based on the proposed method to solve this problem presented in the final section. The method is based on the Sugeno Fuzzy Inference method.

2.3.1.1 Task Transmission Rate Problem Consider a task divider node, and this node sends tasks to a human resource node to which it is assigned. The human node that receives the tasks has a limited capacity. Due to its capacity, the human node tries to avoid data congestion that it has created by rate of the data sent automatically. So, if the task divider's tasks lead to congestion in the human resources node, it will reduce the task transmission rate. If the task transmission rate does not lead to congestion and the waiting time in the human node queue be zero, the human node tries to increase the task division node assignment rate.

This study assumes that task divider nodes send tasks to human resources nodes with three priorities: high priority, medium priority, and low priority. The goal is to reduce the waiting time in line for tasks. For this purpose, a controller is needed to adjust the task submission rate in such a way that the waiting time in the queue is minimized [9]. So, a controller based on a fuzzy inference system has been used to control the rate of sending tasks to human power nodes. The creativity of this controller is described in the proposed fuzzy controller section as follows.

2.3.1.2 Proposed Fuzzy Controller This section has tried to describe the design method of intelligent controller based on fuzzy inference system. In classical set theories, each set is defined as a definite collection of objects, in other words, the classical set has a well-defined feature. For example, a set of real numbers greater than 100 has a welldefined property. We can say with certainty whether this number is greater than 100 or not for any real number. But in general, we cannot talk about the feature of "greatness" as a specific and precise feature. Therefore, these attributes cannot be considered as classic futures.

Many attributes that we deal with in different branches of science, especially humanities, are parts of this category. Then, Fuzzy set theory is a new mathematical format for analyzing these concepts and properties, which is a development in terms of classical sets proposed by Professor Lotfizadeh in 1965 [39]. In concept, Fuzzy Logic is a soft computational method performed in the presence of uncertainties [11, 41]. Now, suppose $X$ is an arbitrary reference set. The characteristic function of any ordinary 
subset $A$ is from $X$ to $\{0,1\}$ that results of which is described in Eq. (9):

$X_{A}(x)= \begin{cases}1 & x \in A \\ 0 & x \notin A\end{cases}$

Now if the vector of the characteristic function is extended from a set of two members $\{0,1\}$ to the interval $[0,1]$, a function is obtained that assigns a number from the interval $[0,1]$ to each $x$ of $\mathrm{X}$, that the function Membership is called A. According to the above definition, $\mathrm{A}$ is no longer an ordinary set, but it calls a fuzzy set. Therefore, a fuzzy set $\mathrm{A}$ is a set which membership degree of it can obtain from $[0,1]$, continuously. If the membership function A denotes by $\mu_{A}(x)$, it turns out that $\mu_{A}(x)$ is a twodimensional function that assigns to each member of $\mathrm{X}$ a number from the interval $[0,1]$ as the degree of membership of that element in the fuzzy set A and is called membership function. The proximity of $\mu_{A}(x)$ to the number 1 indicates that $\mathrm{x}$ belongs more to the fuzzy set $\mathrm{A}$, and conversely. A fuzzy system can have several membership functions for each variable. These functions can take any shape, but these are more common with triangular, trapezoidal, or Gaussian shapes that require the use of a fuzzy controller to optimize fuzzy operations [30].

2.3.1.3 Fuzzy Controller Although it was possible to model many systems in nature until the early two decades, but there was no ability to describe some ambiguous or human systems mathematically. Given that the model of many systems in nature is ambiguous, fuzzy controllers based on fuzzy logic systems are the best option for providing a suitable controller. These controllers are based on IF-Then fuzzy rules and these enter fuzzy input and apply their rules to do the operation. Then, the fuzzy rules are solved using fuzzy inference, and the final fuzzy output is crisped using defuzzification methods.

The defuzzification method is actually converting a fuzzy value to a crisp value. One of the most well-known of these methods is the gravity center method. The two most well-known types of fuzzy controllers are the Mamdani fuzzy controller and the Sugeno fuzzy controller that the most common way these methods use is to set the controller in a forward path in a closed loop system. Of course, output of the process is compared to a reference, and if it is different, the controller applies the required signal to the process based on the existing difference and its control strategy. In general, input or output can have several different signals, multi-input or multi-output systems [31]. Figure 2 shows the components of a fuzzy inference system.

Sugeno issued the Sugeno Fuzzy Controller in 1985 [40]. In this fuzzy output system, each rule is considered a linear function of inputs. Examples of Sugeno fuzzy laws are as follows:

If $x_{1}, A_{1}$ and $y_{1}, B_{1}$ then $\mathrm{z}$ is $f_{1}(x, y)$ and if $x_{n}, A_{n}$ and $y_{n}$, $B_{n}$ then $\mathrm{z}$ is $f_{n}(x, y)$.

In the above rules, $A_{i}$ and $B_{i}$ are fuzzy sets and $x_{i}$ and $y_{i}$ are inputs [32]. Figure 3 shows how Sugeno controller calculates the fuzzy output.

Finally, the definite output is calculated based on the center of a discrete mass. Sugeno fuzzy controller has the ability to model the ability to model the system linearly and can be used in cases where the mathematical model of the system cannot be formulated analytically. Also, due to the output's linearity, it is easy to implement this controller [31].

\section{Case Study and Validation of the Solving Methods}

In this research, we have used two set of data as A and B from [33]. Test set A includes samples with a maximum of 4 sources taken from these two studies' samples. Because MIP results are not available for larger samples. This study only includes a test set of specimens with a maximum of 55 activities. So, we have 509 samples set of the A, and these samples are labeled as a sample set of $A \leq 55$ whose indices show the number of activities. Test set $\mathrm{B}$ includes samples of sets: $B_{10}, B_{20}, B_{40}, B_{100}$ and $B_{200}$ with $10,20,40$, 100 , and 200 activities, respectively, and a maximum of 4 sources. Fündeling and Trautmann [33] generated up to 480 samples in each test set using order strength factorial design of problem parameters, source factor, and source power. However, if the order strength is higher, the priority relationships in the project network will be higher. So, in this study, it has used values of $0.25,0.5$, and 0.75 as order strengths.

The resource factor indicates the number of resources required per activity. Its values are $0.25,0.5,0.75$ and 1 . For example, a value of 0.5 indicates that each activity requires 2 of 4 sources. Resource Strength (RS) also measures resource scarcity by comparing resource demand with resource availability levels. In this study, RS values will be zero, $0.25,0.5$, and 0.75 . Also, lower RS leads to more lakeness. For RS $=0$ there is at least one activity for each resource that may specifically occupy the resource due to the high usage limit of the $\mathrm{q}_{\mathrm{ir}}$ resource, and for $\mathrm{RS}=1$ access to the resource does not limit scheduling. The minimum time interval is also set between 2 and 4 blocks, randomly [33, 34].

In each sample of the problem, all activities require the main source named as $\hat{r}$. In addition, for the independent source $r$, the components of the source function include: 


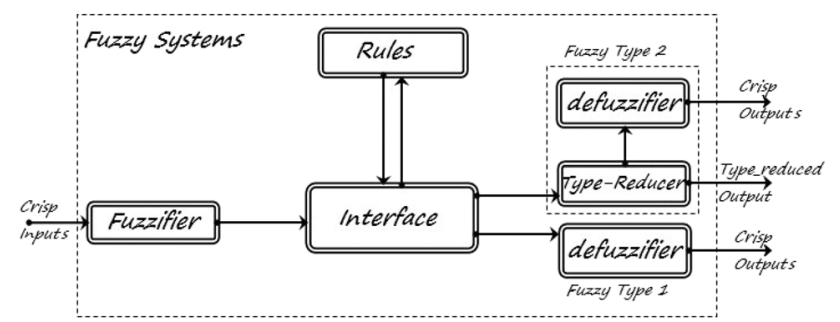

Fig. 2 Components of a fuzzy interference system

$\alpha_{i r}=\left(\bar{q}_{i r}-\underline{q}_{i r}\right) /\left(\bar{q}_{i \hat{r}}-\underline{q}_{i r}\right) \quad$ and $\quad \beta_{i r}=\underline{q}_{i r}-\underline{q}_{i r} \alpha_{i r}$ are used in algorithmic problem solving, according to Naber and Kolisch [34].

\subsection{Comparing the Results of the Proposed Method with the Best Methods in Previous Research}

In this research, the proposed method will be compared with the following methods from previous research:

- Self-Adapted Genetic Algorithm (SGA) [34].

- Parallel Random Heuristic Modeling (PRS): Creates an activity list by random pattern modeling and generates scheduling with standard parallel SGS [35].

- Heuristic Serial Random Modeling (SRS): This is the same as PRS but uses standard serial SGS.

- MIP: Known as the reference for commercial solvents using the FP-DT3 of PIM model. The best solution obtained after $2 \mathrm{~h}$ is considered as the answer [34].
- HM: This method was proposed by Tritschler et al. [9] which is a metaheuristic method to solve a research problem.

In addition, to analyze the effect of HM components on the quality of the solution, three HM variables were compared, and the results were compared with the proposed method.

- GA-FSGS: FSGS without VNS is embedded in GA. This combination has been used to evaluate the effect of VNS [35].

- MP-FSGS: To evaluate the effect of GA, FSGS is used in a multi-pass method. Using FSGS, this method generates a large amount of scheduling and selects the best value for the objective function. We produce three $\lambda$ with the LPF, MTS and MWR priority rules and $\rho$ and $\sigma$ are zero. We select the remaining $\lambda$ using random activity selection and $\rho$ and $\sigma$ randomly within the given boundary range [33].

- GA-SGS: GA only works in $\lambda$ and is combined with standard parallel SGS. This combination has been used to evaluate FSGS.

The methods mentioned above are compared based on the maximum number of produced scheduling $(\Omega)$ per sample number. In addition, if the project execution time be $T_{\min }$, then the optimal scheduling is obtained, the calculations are completed and the computation time required for each method is expressed as a single CPU time [36-]. However, the proposed method of present study has been run in MATLAB software using a computer with a 4.3 GHz Intel Core i7-7700 k CPU with $16 \mathrm{~GB}$ of RAM.

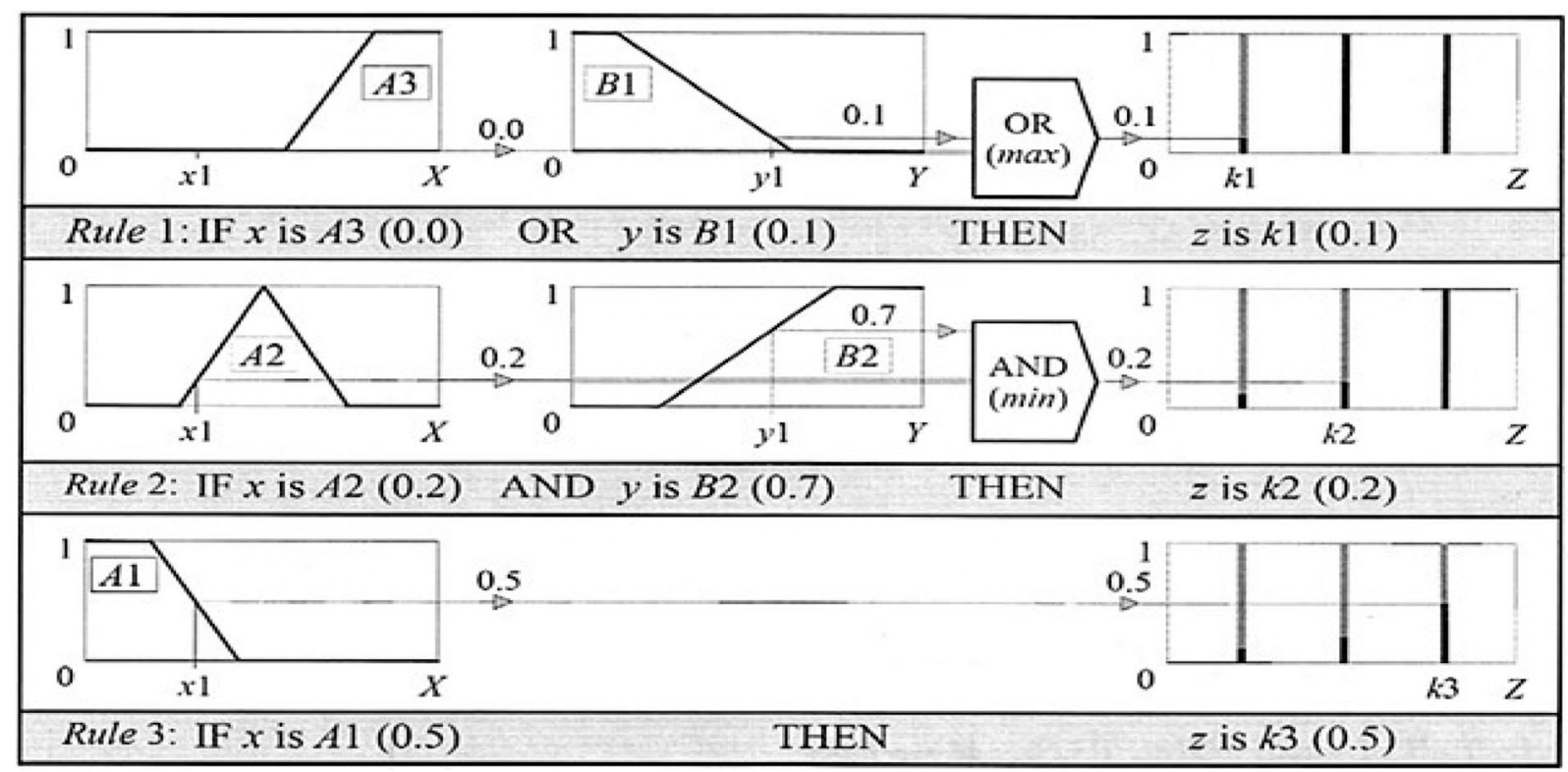

Fig. 3 How Sugeno controller calculates the fuzzy output 
Table 2 Mean deviation as a percentage of the best MIP $\left(\Delta_{\text {mip }}\right)$ and $T \min \left(\Delta_{\mathrm{lb}}\right)$ solution

\begin{tabular}{|c|c|c|c|c|c|c|c|c|c|c|}
\hline \multirow[t]{2}{*}{ Set $/ \Omega$} & \multicolumn{2}{|c|}{ Proposed Method } & \multicolumn{2}{|c|}{ Tritschler et al. [9] } & \multicolumn{2}{|l|}{ SGA } & \multicolumn{2}{|l|}{ PRS } & \multicolumn{2}{|l|}{ SRS } \\
\hline & $\Delta_{\text {mip }}$ & $\Delta_{\mathrm{lb}}$ & $\Delta_{\text {mip }}$ & $\Delta_{\mathrm{lb}}$ & $\Delta_{\text {mip }}$ & $\Delta_{\mathrm{lb}}$ & $\Delta_{\text {mip }}$ & $\Delta_{\mathrm{lb}}$ & $\Delta_{\text {mip }}$ & $\Delta_{\mathrm{lb}}$ \\
\hline $\mathrm{A} \leq 55$ & -0.73 & 5.52 & 0.03 & 5.60 & 2.12 & 7.82 & 2.64 & 8.41 & 3.89 & 9.83 \\
\hline 1000 & 0.11 & 4.73 & 0.64 & 6.27 & 2.52 & 8.30 & 3.26 & 9.09 & 4.66 & 10.68 \\
\hline 5000 & -0.54 & 3.76 & 0.01 & 5.58 & 2.13 & 7.83 & 2.67 & 8.45 & 3.95 & 9.86 \\
\hline 15000 & -0.56 & 4.68 & -0.24 & 5.30 & 1.95 & 7.63 & 2.37 & 8.10 & 3.57 & 9.47 \\
\hline 25000 & -1.07 & 3.96 & -0.27 & 5.25 & 1.88 & 7.53 & 2.26 & 7.98 & 3.40 & 9.28 \\
\hline B10 & 0.16 & 3.73 & 0.24 & 5.40 & 1.31 & 6.57 & 1.55 & 6.81 & 1.59 & 6.88 \\
\hline 1000 & -0.51 & 4.12 & 0.44 & 5.62 & 1.34 & 6.61 & 1.56 & 6.83 & 1.62 & 6.91 \\
\hline 5000 & -0.10 & 3.94 & 0.22 & 5.38 & 1.30 & 6.56 & 1.55 & 6.82 & 1.58 & 6.87 \\
\hline 15000 & -0.24 & 3.63 & 0.15 & 5.31 & 1.30 & 6.56 & 1.55 & 6.81 & 1.58 & 6.87 \\
\hline 25000 & -0.85 & 4.60 & 0.14 & 5.28 & 1.30 & 6.56 & 1.55 & 6.81 & 1.58 & 6.87 \\
\hline B20 & -0.64 & 3.81 & 0.28 & 4.24 & 0.75 & 4.77 & 1.28 & 5.34 & 1.31 & 5.40 \\
\hline 1000 & -0.21 & 3.31 & 0.60 & 4.60 & 0.86 & 4.90 & 1.63 & 5.73 & 1.70 & 5.84 \\
\hline 5000 & -0.41 & 3.48 & 0.27 & 4.23 & 0.75 & 4.78 & 1.31 & 5.37 & 1.30 & 5.39 \\
\hline 15000 & -0.05 & 2.79 & 0.15 & 4.10 & 0.70 & 4.71 & 1.12 & 5.16 & 1.15 & 5.23 \\
\hline 25000 & -0.53 & 3.07 & 0.10 & 4.04 & 0.69 & 4.70 & 1.07 & 5.10 & 1.09 & 5.16 \\
\hline B40 & -2.86 & 3.05 & -1.88 & 4.08 & -1.73 & 4.29 & -0.05 & 6.21 & 0.32 & 6.74 \\
\hline 1000 & -2.53 & 3.02 & -1.66 & 4.35 & -1.53 & 4.55 & 0.49 & 6.84 & 0.93 & 7.44 \\
\hline 5000 & -2.69 & 3.74 & -1.88 & 4.09 & -1.72 & 4.31 & -0.01 & 6.26 & 0.42 & 6.85 \\
\hline 15000 & -2.54 & 3.39 & -1.98 & 3.96 & -1.82 & 4.18 & -0.29 & 5.94 & 0.04 & 6.41 \\
\hline 25000 & -2.56 & 3.70 & -2.10 & 3.93 & -1.85 & 4.13 & -0.4 & 5.81 & -0.09 & 6.26 \\
\hline B100 & & 2.10 & & 3.94 & & 4.05 & & 7.15 & & 8.09 \\
\hline 1000 & & 3.65 & & 4.07 & & 4.21 & & 7.68 & & 8.86 \\
\hline 5000 & & 3.52 & & 3.98 & & 4.08 & & 7.21 & & 8.14 \\
\hline 15000 & & 3.72 & & 3.89 & & 3.97 & & 6.92 & & 7.84 \\
\hline 25000 & & 2.21 & & 3.87 & & 3.93 & & 6.80 & & 7.71 \\
\hline B200 & & 1.72 & & 3.41 & & 3.55 & & 7.12 & & 8.20 \\
\hline 1000 & & 1.60 & & 3.46 & & 3.65 & & 7.53 & & 8.67 \\
\hline 5000 & & 2.04 & & 3.40 & & 3.57 & & 7.16 & & 8.27 \\
\hline 15000 & & 2.30 & & 3.39 & & 3.51 & & 6.95 & & 7.98 \\
\hline 25000 & & 1.82 & & 3.39 & & 3.48 & & 6.83 & & 7.88 \\
\hline Sum & -0.97 & 3.41 & -0.33 & 4.46 & 0.63 & 5.20 & 1.37 & 6.86 & 1.81 & 7.55 \\
\hline
\end{tabular}

\subsection{Setting the Parameters}

One of the proposed method features is that there is no need to set the parameters, manually for each type of HRA problem. In this study, to adapt GA to the problems with different sizes, the group size is set according to the min $(10 \cdot \mathrm{n}, 400)$ function, and used the number of activities $\mathrm{n}$, and the transformation rate for the activity list $\mathrm{p} \lambda=5 \%$ and also $p_{\sigma}=0.5 \%$ and $p_{\rho}=5 \%$. Besides, the interaction between GA and VNS is simplified by adjusting the number of schedules as a termination criterion based on the size of the problem. Also, for the VNS schedule limit is set the value of $\Omega_{\mathrm{VNS}}=\Omega \cdot \max (\mathrm{n} / 200,0.25)$ and for the GA schedule limit is set the value of $\Omega_{\mathrm{GA}}=\Omega-\Omega_{\mathrm{VNS}}$. Also, in the VNS it has used 1000 unimproved schedules for the proposed solution, 200 generated schedules for the neighborhood, the maximum 5 neighborhood, and 5 longest route in the activity selection. For GSA, the min group's size $(5 \cdot n, 200)$ was used, and the list of activities in the initial group was created as HM. The SGS flag is randomly set with the same probability for serial or parallel, and the conversion rates are set to $5 \%$ for both of the activity list and the SGS flag [37]. 
Table 3 Average time to generate 1000 schedules per second

\begin{tabular}{llllllll}
\hline Set & Proposed Method & Tritschler et al. [9] & GA & VNS & SGA & PRS & SRS \\
\hline A $\leq 55$ & 0.0006 & 0.14 & 0.11 & 0.24 & 0.05 & 0.06 & 0.02 \\
B10 & 0.0002 & 0.08 & 0.08 & 0.17 & 0.04 & 0.04 & 0.02 \\
B20 & 0.0010 & 0.2 & 0.18 & 0.28 & 0.09 & 0.09 & 0.05 \\
B40 & 0.0084 & 0.5 & 0.38 & 0.65 & 0.21 & 0.21 & 0.1 \\
B100 & 0.0146 & 1.55 & 1.19 & 2.72 & 0.84 & 0.69 & 0.31 \\
B200 & 0.019 & 4.44 & 3.58 & 9.08 & 2.61 & 1.85 & 0.7 \\
Sum & 0.01 & 1.15 & 0.92 & 2.19 & 0.64 & 0.49 & 0.2 \\
\hline
\end{tabular}

\subsection{Research Findings}

\subsubsection{Computational Results}

In this research, when we talk about statistical significance, it refers to the significance level of $\alpha=0.05$ confirmed in the Kruskal-Wallis, and single-sided analysis of variable or Mann-Whitney U test.

\subsubsection{Quality of Solution}

Table 2 shows the mean relative deviation of the mean $\Delta_{\text {mip }}$ obtained from the best MIP solution and comparative mean deviation of $\Delta_{\mathrm{lb}}$ from the lower bound of the $\mathrm{T}_{\min }$ project execution time. Optimal MIP solutions for all samples are obtained from the $\mathrm{B} 10$ set, while for sets: $\mathrm{B}_{20}, \mathrm{~B}_{40}$, and $\mathrm{A} \leq 55$, all solutions are not optimal. For each sample set, average results for schedule limits $(\Omega)$ are given four schedules amount includes 1000, 5000, 15,000, and 25,000, and the average result is highlighted throughout all scheduling limits. The last row of the table shows the overall results for all sample sets and scheduling limits. The differences between the methods are statistically significant in all rows.

Based on the results presented in Tables 2, the proposed method leads to better results than other statistically significant methods. The advantage of Tritschler et al. [9] is the maximum value in the B10, while all MIPs are solved to optimize and $\Delta_{\text {mip }}$ is equal to the optimization distance. In Tritschler et al. [9], for 25,000 scheduling, with the optimization distance of $14 \%$, it is about 9 times less than the same value in other methods.

Research by Tritschler et al. [9] improves with an increase of $\Omega$ from 15,000 to 25,000 while other methods remain the same. For the average $\mathrm{A} \leq 55$ and B 40 datasets, $\mathrm{HM}$ finds some of the best new solutions. For larger B100 and B200 datasets, HM and SGA perform better heuristic random modeling, clearly. The proposed method also provides better results than the research of Tritschler et al. [9] for all cases.

\subsubsection{Computational Time}

Computing the average time in seconds requires generating 1000 scheduling for each problem (based on the average of the 25,000 scheduling generated) listed in Table 3 . Although this is not completely linear, but it still works well. Tritschler et al. [9] computational time increases as a fixed factor equal to 2.5 through doubling the number of activities when considering the range of 10 up to 100 activities. For 200 activities, the factor slowly increases to 2.86. Because of repetitive production and analysis of source trends in selected activity, VNS of Tritschler et al. [9] requires more time. As VNS does not run in all solutions, its effect on HM computation time is small. The key factor for computation time is the used SGS, as the FSGS runs more complex operations to determine the source value compare to other SGSs. The proposed method's computational time is almost 100 times better compared to any case study that has issued in literature review of Tritschler et al. [9]. This was because of strength in the used fuzzy system, and extended in the GWO algorithm added self-regulating attributes to the basic algorithm.

\subsection{The Sample Parameters}

The effect of sample parameters on the quality of the solution is evaluated. Table 4 and Fig. 4 show the $T_{\text {min }}$ distance for different amounts of ordinarily strength (OS), resource factor (RF), resource strength (RS), and minimum block length. Table 4 shows the averages for each of the four scheduling limits $\Omega$ are plotted for the B10 to B200. According to Fündeling and Trautmann [35] results ordinarily, strength has an irreversible different amounts of ordinarily strength effect on the quality of the solution. The effect of the minimum block length is small, too. Resource strength and resource factors have a significant effect on the quality of the solution.

Figure 5 shows the ratio of distance to $T_{\min }$ mean along with all four scheduling limits of $\Omega$. The difference in results is statistically significant in all rows of the table. By considering pairwise comparisons for overall results in the 
Table 4 Average distance up to $\mathrm{T}_{\text {min }}$ based on percentage

\begin{tabular}{llllll}
\hline Set & Proposed Method & Tritschler et al. [9] & GA-FSGS & MP-FSGS & GA-SGS \\
\hline A $\leq 55$ & 5.26 & 5.6 & 5.69 & 6.92 & 7.88 \\
B10 & 4.43 & 5.4 & 5.41 & 5.6 & 6.85 \\
B20 & 3.87 & 4.24 & 4.28 & 5.02 & 5.09 \\
B40 & 3.72 & 4.08 & 4.17 & 5.84 & 4.54 \\
B100 & 3.32 & 3.94 & 4.09 & 5.23 & 4.16 \\
B200 & 2.78 & 3.41 & 3.58 & 4.24 & 3.69 \\
Sum & 3.89 & 4.46 & 4.55 & 5.49 & 5.39 \\
\hline
\end{tabular}

Table 5 Solution quality for FRCPSP with discrete sources

\begin{tabular}{|c|c|c|c|c|c|c|}
\hline \multirow[t]{2}{*}{ Sample } & \multicolumn{2}{|c|}{ Proposed Method } & \multicolumn{2}{|l|}{$\mathrm{HM}$} & \multicolumn{2}{|c|}{ GA-FSGS } \\
\hline & $\Delta_{\text {mip }}$ & $\Delta_{\mathrm{lb}}$ & $\Delta_{\text {mip }}$ & $\Delta_{\mathrm{lb}}$ & $\Delta_{\text {mip }}$ & $\Delta_{\mathrm{lb}}$ \\
\hline B10 & 0.23 & 5.68 & 0.29 & 5.77 & 0.31 & 5.79 \\
\hline 1000 & 0.47 & 6.04 & 0.55 & 6.06 & 0.58 & 6.09 \\
\hline 5000 & 0.21 & 5.65 & 0.26 & 5.47 & 0.28 & 5.76 \\
\hline 15,000 & 0.17 & 5.59 & 0.18 & 5.64 & 0.19 & 5.66 \\
\hline 25,000 & 0.18 & 5.62 & 0.18 & 5.65 & 0.19 & 5.66 \\
\hline B20 & -0.08 & 4.52 & -0.04 & 4.56 & -0.02 & 4.58 \\
\hline 1000 & 0.25 & 4.84 & 0.26 & 4.89 & 0.27 & 4.91 \\
\hline 5000 & -0.04 & 4.47 & -0.03 & 4.57 & -0.02 & 4.58 \\
\hline 15,000 & -0.24 & 4.33 & -0.17 & 4.41 & -0.14 & 4.44 \\
\hline 25,000 & -0.31 & 4.34 & -0.22 & 4.36 & -0.19 & 4.39 \\
\hline B40 & -2.01 & 4.44 & -2.01 & 4.47 & -2.01 & 4.47 \\
\hline 1000 & -1.74 & 4.86 & -1.67 & 4.86 & -1.77 & 4.76 \\
\hline 5000 & -2.06 & 4.43 & -2.04 & 4.43 & -2.02 & 4.45 \\
\hline 15,000 & -2.15 & 4.26 & -2.14 & 4.31 & -2.10 & 4.36 \\
\hline 25,000 & -2.28 & 4.18 & -2.18 & 4.26 & -2.14 & 4.31 \\
\hline B100 & & 4.14 & & 4.21 & & 4.34 \\
\hline 1000 & & 4.29 & & 4.34 & & 4.42 \\
\hline 5000 & & 4.16 & & 4.20 & & 4.34 \\
\hline 15,000 & & 4.17 & & 4.17 & & 4.30 \\
\hline 25,000 & & 4.13 & & 4.15 & & 4.29 \\
\hline Sum & -0.63 & 4.71 & -0.58 & 4.75 & -0.57 & 4.79 \\
\hline
\end{tabular}

last row, GA-FSGS generates better results than MP-FSGS and GA-SGS with clear statistical signs. This identifies the positive effects of GA (between GA-FSGS and MP-FSGS) and FSGS (between GA-FSGS and GA-SGS), clearly. Trichler et al. [9] advances are statistically insignificant compared to GA-FSGS when considering all samples. However, this analysis includes examples on which VNS has not been implemented. The differences are quite noticeable between the proposed method and other methods, and the proposed method shows basic improvement in the solutions, too.

\subsubsection{Dependence on Fuzzy-RCPSP with Discrete Sources}

Despite continuous sources that are examined in this study, there are empirical cases whose number of sources is constrained, such as those stated in Fündeling and Trautmann [35] and Baumann et al. [38]. This section 

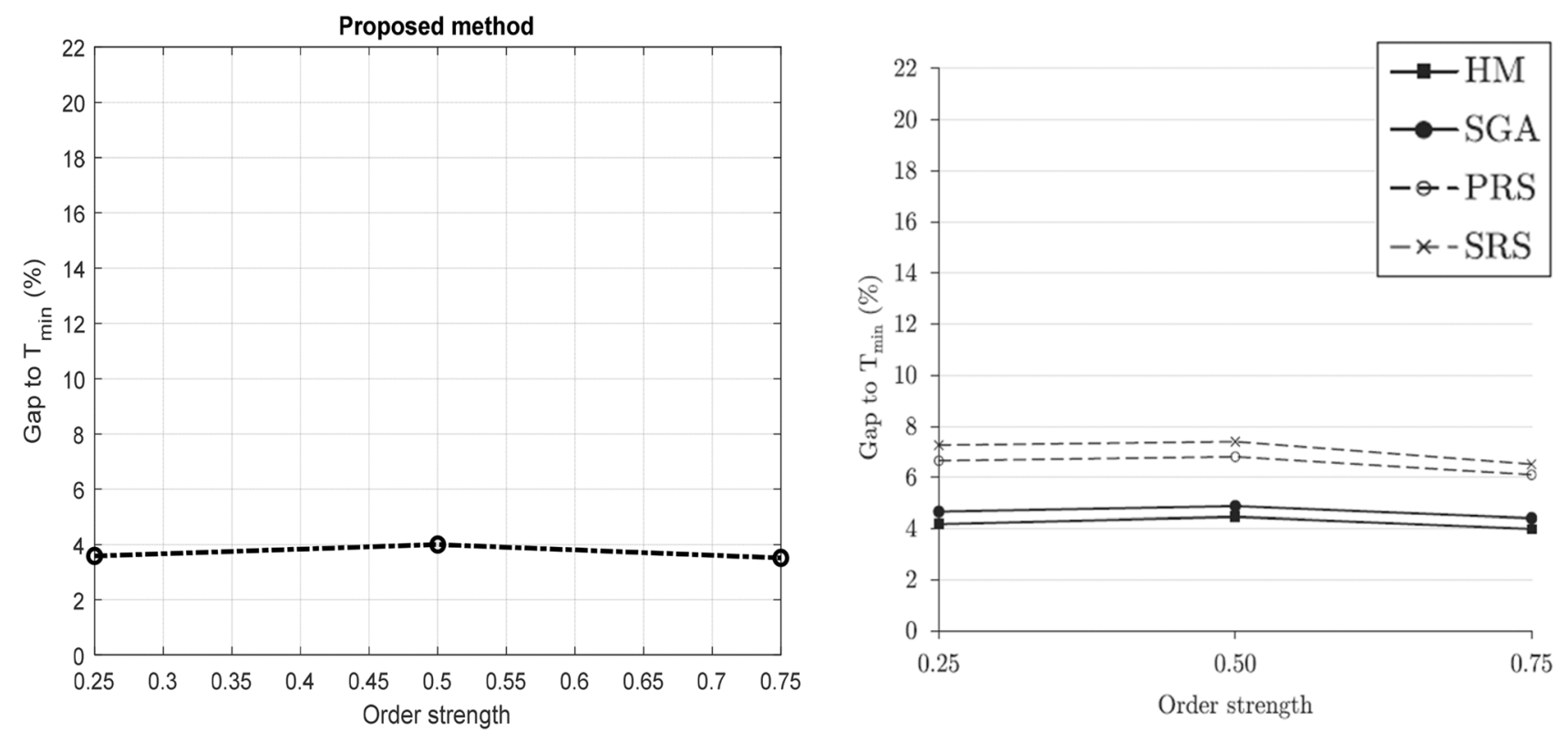

Fig. 4 Output of the proposed method and Tritschler et al. [9] for $T_{\min }$ distance for different values of Ordinarily Strength
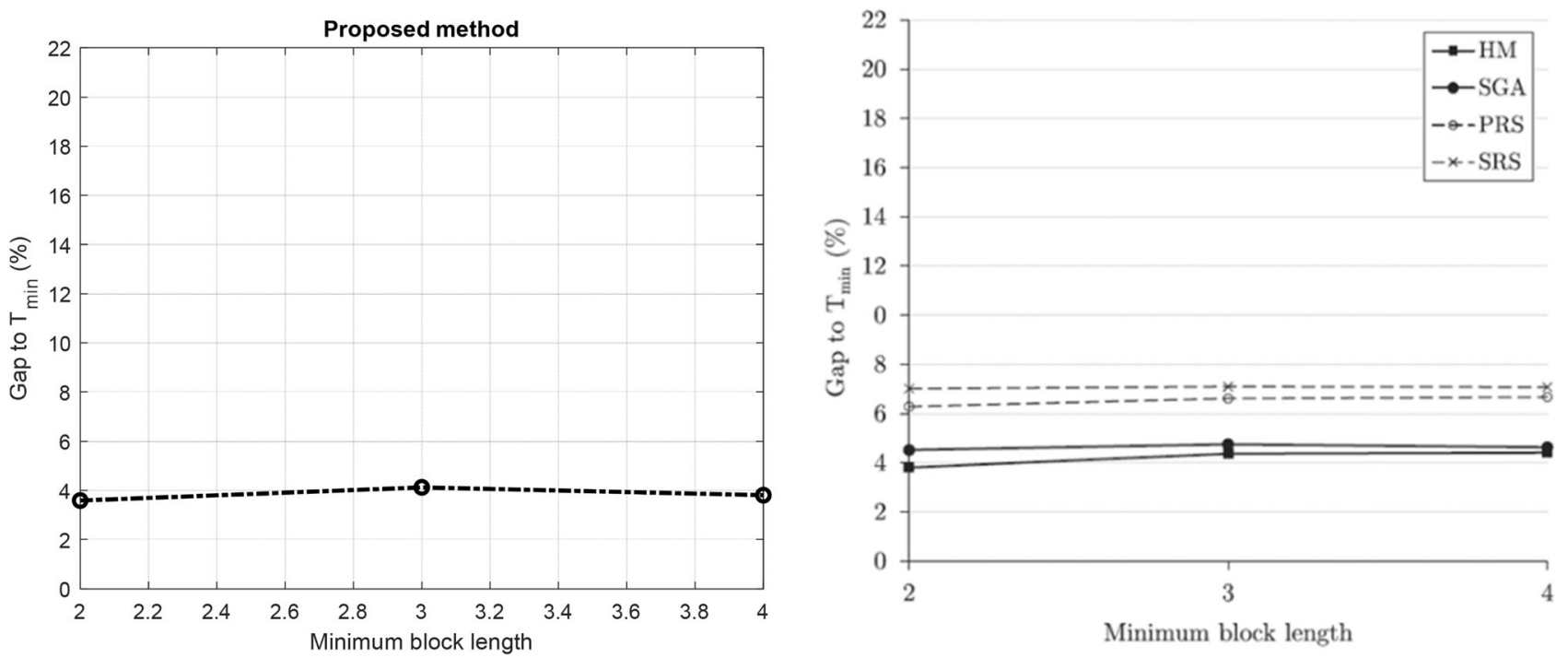

Fig. $5 T_{\min }$ Distance for different values of minimum block length through the proposed method and Tritschler et al. [9]

demonstrates how the proposed method can also solve discrete resource problems under the fuzzy approach. It is important to note that despite the distribution with discrete sources, the computational results of discrete sources cannot be compared with those in the Fündeling and Trautmann [35] and Baumann et al. [38]. Prior to these problems, they placed the exact and detailed resources in the exact amount requested by each activity, while our problem allows resources to be allocated at least the required amount for each activity and blends in with the real environment, thus gaining a fuzzy mood. In other words, more resources may be allocated to activities, while the duration of global projects can be reduced, while trying to cover the minimum required block length.

The attribute value of $\mathrm{q}_{\text {irt }}$ is the main variable that differentiates between discrete and continuous sources. 
Therefore, we converted the $\mathrm{q}_{\text {irt }}$ continuous value to the integer value by rounding to up or down values till it can examine the required resources by an activity and the access limits of each resource, respectively. We performed the discrete resource-guided implementations that the results calculated from solving experimental sets include: B10, B20, B40, and B100, which are issued in Table 5. It should be noted that VNS are related to these sample sets in that, and they maintain the stated integer conditions of resource and boundary requirements. A very small increase is expected in execution times in the rounding operation for discrete sources.

Table 5 shows quality of solutions in the mean deviation stages of the best MIP DR solution. Also, it reports the duration of $\mathrm{T}_{\min }$ in project for both HM and GA-FSGS from the lower bound. While reference $\mathrm{T}_{\min }$ values remain unaffected, the best MID DR solutions were obtained by solving the best FP-DT3 model compare to Naber and Kolisch [34], although it was imposed integrating on all the source variables.

Despite the fact that the MIP DR model is generally more difficult to solve than the CR model, it was found that all MIP solutions are optimal for the B10 test set. It is used instead of the MIP reference value for those cases that the MIPs were not optimally solved. Also, for this item, modified discrete source test and errored the best project execution time or the upper limit of the project execution time obtained from the Neber and Kolisch [34]. The average distance $\Delta_{\mathrm{lb}}$ from the discrete sources is, as expected, slightly greater than the continuous sources for all experimental sets because of the increasing integrity of the source variables. The mean $\Delta_{\text {mip }}$ distance of discrete sources is slightly better than the continuous sources in all expected B10 experimental sets.

This optimal output is because of the poor quality of the MIP solution at limited execution times. Despite the applicability of the proposed method, the method of Tritschler et al. [9], and the GA-FSGS concerning discrete sources, it should be emphasized that these methods are designed for continuous sources. Further adaptation of these methods for future studies is suggested to improve the quality of the solution for FRCPSP with discrete sources as a combination of several types of sources. Table 5 shows the proposed solution's quality in the fuzzy mode for different modes of the problem.

According to the results, the proposed method's results have issued better results compared to the advanced algorithms and the methods that are proposed or used in previous research. This study considered the HRA problem's preconditions under environmental realities and with a real range of changes, and the proposed method solved the HRA problem with a flexible structure in both normal and critical conditions. The results confirmed the superiority of the proposed method over previous research.

\section{Conclusion}

In this study, the authors tried to use a new framework to continuously and comprehensively solve HRA problems (In normal and critical conditions under variability of goals and organizational conditions). For this, a developed GWO algorithm was used. In this study, the mathematical model of the HRA problem was first proposed, and then, the GWO algorithm was developed using the Sugeno fuzzy inference model to solve the problem. The problem was solved with different data from previous researches, and the results were compared with those researches. In a conceptual context, the concept of separating the performing tasks page was used in this research. For this purpose, a two-layer framework was proposed to perform HRA in two layers, continuously and under uncertainty conditions. It was to avoid the congestion of tasks in the receiving nodes and load imbalance on the manpower nodes. It received tasks in a way that prevented overcrowding in the task transmission rate adjustment layer and task receiving nodes. Simultaneously, in the resource allocation layer the available human resources were optimally allocated using a developed GWO algorithm after evaluating the current situation. In fact, to improve the overall search of the standard GWO, three optimal solutions from the current population of solutions were used, which included: the best optimal population solution $\alpha$, and the second and third optimal solutions were $\beta$ ' and $\delta$,' respectively. Also, these three optimal solutions cause the search space to be expanded and the overall search in the algorithm, because of the change in their values each time the algorithm is run. Finally, combination of GWO algorithm and Sugeno Fuzzy Inference Model is to create new self-regulating algorithm that occurs for the first time in developing local search algorithms.

This study used two samples A and B test sets of Vanhoucke [10]. Also, the present study's proposed method was compared to best methods of HRA in the previous research based on the maximum number of produced schedules per problem sample $(\Omega)$. It was also tried to make the proposed method's termination criteria in solving the HRA problem be reachable. Since the best research in solving the HRA problem was Alvarez-Valdes et al. [8] and Tritschler et al. [9], the finding showed that the proposed method had better results compare to all previous methods, and the quality of the solutions have been better in this research. As future research suggests, combining the newly developed algorithm with Artificial Intelligence capabilities to authomize automate the running 
and algorithm behavior completely. It can also be a progress advantage to compare the results of the AI algorithms with the proposed algorithm in HR-Tech categories too.

\section{References}

1. Dabirian, S.H., Abbaspour, S., Khanzadi, M., Ahmadi, M.: Dynamic modelling of human resource allocation in construction projects. Int. J. Constr. Manag. (2019). https://doi.org/10.1080/ 15623599.2019.1616411

2. Arias, M., Munoz-Gama, J., Sepúlveda, M.: Towards a taxonomy of human resource allocation criteria. International Conference on Business Process Management, BPM 2017: Business Process Management Workshops. 308, 475-483 (2018). https://doi.org/ 10.1007/978-3-319-74030-0_37

3. Erasmus, J., Vanderfeesten, I., Traganos, K., Jie-A-Looi, X., Kleingeld, A., Grefen, P.: A method to enable ability-based human resource allocation in business process management systems. Lecture Notes Bus. Inf. Process. The Practice of Enterprise Modeling. PoEM 2018. Lecture Notes in Business Information Processing, 335, 37-52 (2018). https://doi.org/10.1007/978-3030-02302-7_3

4. Arias, M., Saavedra, R., Marques, M.R., Munoz-Gama, J., Sepúlveda, M.: Human resource allocation in business process management and process mining: a systematic mapping study. Manag. Decis. 56(2), 376-405 (2018)

5. Yua, L., Zhang, C., Yang, H., Miao, L.: Novel methods for resource allocation in humanitarian logistics considering human suffering. Comput. Ind. Eng. 119, 1-20 (2018)

6. Ballesteros-Pérez, P., Ting Phu, F.T., Mora-Melià, D.: Human resource allocation to multiple projects based on members' expertise, group heterogeneity, and social cohesion. J. Constr. Eng. Manag. 145(2), 110 (2019). https://doi.org/10.1061/ (ASCE)CO.1943-7862.0001612

7. Yousefi, M., Yousefi, M.: Human resource allocation in an emergency department: a meta-model-based simulation optimization. Kybernetes 49(3), 779-796 (2019)

8. Alvarez-Valdés, R., Crespo, E., Tamarit, J.M., Villa, F.: GRASP and path relinking for project scheduling under partially renewable resources. Eur. J. Oper. Res. 189(3), 1153-1170 (2008)

9. Tritschler, M., Naber, A., Kolisch, R.: A hybrid metaheuristic for resource-constrained project scheduling with flexible resource profiles. Eur. J. Oper. Res. 262(1), 262-273 (2017)

10. Vanhoucke, M.: Setup times and fast tracking in resource-constrained project scheduling. Comput. Ind. Eng. 54(5), 1062-1070 (2008)

11. Kwak, W., Shi, Y., Jung, K.: Human resource allocation in a CPA firm: a fuzzy set approach. Rev. Quant. Financ. Acc. 20, 277-290 (2003)

12. Saaty, L.T., Peniwati, K., Shang, S.J.: The analytic hierarchy process and human resource allocation: half the story. Math. Comput. Model. 46(7-8), 1041-1053 (2007)

13. Lin, CH-L., Gen, M.: Multi-criteria human resource allocation for solving multistage combinatorial optimization problems using multi-objective hybrid genetic algorithm. Expert Syst. Appl. 34(4), 2480-2490 (2008).

14. Estellon, B., Gardi, F., Nouioua, K.: High-performance local search for task scheduling with human resource allocation. Lecture Notes Comput. Sci. 1, 5752 (2009). https://doi.org/10.1007/ 978-3-642-03751-1_1

15. Filho, F.C., Aparecid, D., Roch, R., GuimarãesFernandes Costa, M., Albuquerque Pereir, W.C.: Using Constraint Satisfaction
Problem approach to solve human resource allocation problems in cooperative health services. Expert Syst. Appl. 39(1), 385-394 (2012)

16. Pérez, P., González-Cruz, M.C., Diego, M.F.: Human resource allocation management in multiple projects using sociometric techniques. Int. J. Project Manage. 30(8), 901-913 (2012)

17. Cabanillas, C., García, J.M., Resinas, M., Ruiz, D., Mendling, J., Ruiz-Cortés, A.: Priority-based human resource allocation in business processes. Lecture Notes Comput. Sci. 1, 8274 (2013). https://doi.org/10.1007/978-3-642-45005-1_26

18. Chien, T.H., Lin, Y.I., Tien, K.W.: Agent-based negotiation mechanism for multi-project human resource allocation. J. Ind. Prod. Eng. 30(8), 518-527 (2013)

19. Stylianou, C., Andreou, A.S.: Human resource allocation and scheduling for software project management. Software Project Management in a Changing World. Springer, Berlin, Heidelberg, 73-106 (2014). https://doi.org/10.1007/978-3-642-55035-5_4

20. Ponsteen, A., J.Kusters, R.: Classification of human- and automated resource allocation approaches in multi-project management. Procedia Soc. Behav. Sci. 194, 165-173 (2015).

21. Park, J., Seo, D., Hong, G., Shin, D., Hwa, J., Ba, D.H.: Human resource allocation in software project with practical considerations. Int. J. Software Eng. Knowl. Eng. 25(1), 5-26 (2015)

22. Wibisono, A., Nisafani, A.S., Bae, H., Park, Y.J.: On-the-fly performance-aware human resource allocation in the business process management systems environment using Naïve Bayes. Lecture Notes Bus. Inf. Process. 1, 219 (2015). https://doi.org/10. 1007/978-3-319-19509-4_6

23. Roque, L., Araújo, A.A., Dantas, A., Saraiva, R., Souza, J.: Human resource allocation in Agile Software projects based on task similarities. Lecture Notes Comput. Sci. 1, 9962 (2016). https://doi.org/10.1007/978-3-319-47106-8_25

24. Lilia, Z.H.: An inverse optimization model for human resource allocation problem considering competency disadvantage structure. Procedia Comput. Sci. 112, 1611-1622 (2017)

25. Bouajaja, S., Dridi, N.: A survey on human resource allocation problem and its applications. Oper. Res. Int. J. 17, 339-369 (2017)

26. Aviso, K.B., Cayamanda, C.D., Mayol, A.P.: Optimizing human resource allocation in organizations during crisis conditions: a P-graph approach. Process Integ. Optim. Sustain. 1, 59-68 (2017)

27. Arias, M., Munoz-Gama, J., Sepúlveda, J., Miranda, J.C.: Human resource allocation or recommendation based on multi-factor criteria in on-demand and batch scenarios. Eur. J. Ind. Eng. (2018). https://doi.org/10.1504/EJIE.2018.092009

28. Xiao, L.: Optimal allocation model of enterprise human resources based on particle swarm optimization. International Conference on Computer Information and Big Data Applications (CIBDA), China, (2020). https://doi.org/10.1109/CIBDA50819.2020.00063.

29. Khanizad, R., Montazer, G.A.: A model for optimal allocation of human resources based on the operational performance of organisational units by multi-agent systems. Int. J. Oper. Res. (2021). https://doi.org/10.1504/IJOR.2021.111952

30. Aslinezhad, M., Malekijavan, A., Abbasi, P.: Adaptive neurofuzzy modeling of a soft finger-like actuator for cyber-physical industrial systems. Journal of Supercomputing (2020). https://doi. org/10.1007/s11227-020-03370-3

31. Marimuthu, P., Perumal, V., Vijayakumar, V.: OAFPM: optimized ANFIS using frequent pattern mining for activity recognition. Journal of Supercomputing 75, 5347-5366 (2019)

32. Yan, F.: Gauss interference ant colony algorithm-based optimization of UAV mission planning. J. Supercomput. 76, 1170-1179 (2020)

33. Fündeling, C.U., Trautmann, N.: A priority-rule method for project scheduling with work-content constraints. Eur. J. Oper. Res. 203(3), 568-574 (2010) 
34. Naber, A., Kolisch, R.: MIP models for resource-constrained project scheduling with flexible resource profiles. Eur. J. Oper. Res. 239(2), 335-348 (2014)

35. Kolisch, R., Hartmann, S.: Experimental investigation of heuristics for resource-constrained project scheduling: An update. Eur. J. Oper. Res. 174(1), 23-37 (2006)

36. Van Peteghem, V., Vanhoucke, M.: An experimental investigation of metaheuristics for the multi-mode resource-constrained project scheduling problem on new dataset instances. Eur. J. Oper. Res. 235(1), 62-72 (2014)

37. Hartmann, S.: A self-adapting genetic algorithm for project scheduling under resource constraints. Nav. Res. Logist. 49(5), 433-448 (2002)

38. Baumann, P., Fündeling, C.U., Trautmann, N.: The resourceconstrained project scheduling problem with work-content constraints. Handb. Project Manag Sched 1, 533-544 (2015)

39. Zadeh, L.A.: Fuzzy sets. Inf. Control 8(3), 338-353 (1965)

40. Sugeno, M.: Industrial Applications of Fuzzy Control. Elsevier Science Ltd; First Edition, Japan. ISBN-13: 978-0444878298 (1985).

41. Özdilek, Ü.: Property valuation based on Choquet integral. Comp. Appl. Math. 39, 98 (2020). https://doi.org/10.1007/ s40314-020-1122-z

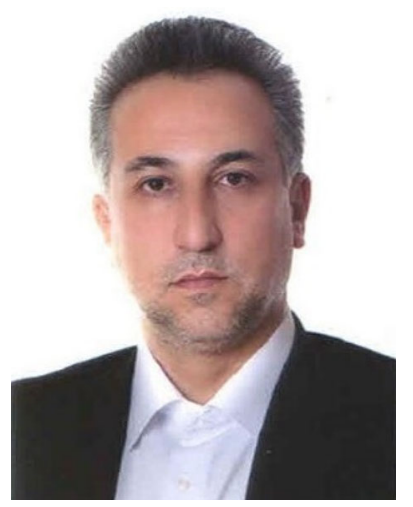

Dr. Hamid-Reza Seifi was awarded his BSc in Power Engineering at Shahid-Abbaspour Institute in 1998. He was graduated in System Management at Industrial Organization in 2008. He has started PhD degree at Amirkabir University since 2017. He is well-known senior manager at Iran in private section. He has experienced over than 34 top positions in this section up to now in Iran. He is representative of ILO in Iran during last 12 years ago up to now. He has held workshops in the field of management, entrepreneurship, enterprise engineering, and HRM in many private and public organizations.He is in charge of most important research team of organization optimization in Iran since 2019 as a national group to move Iranian organization to new HR-Tech/HT organizations.Also, he has published many original and review papers in Iranian journals during last 8 years ago up to now.

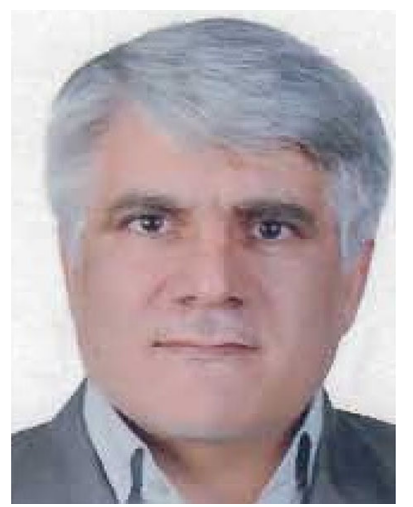

Dr. Naser Shams Gharneh was awarded his BSc in Textile Engineering at Amirkabir University of Technology in 1978. In 1980 he was one of the committee members to organise Jahad Daneshgahi at Amirkabir university. Over the period from 1980 to 1986 , he worked in different department at Jahad such as cultural, educational and research departments. In 1986, based on his experiences in student affairs he was appointed as the vice chancellor for student affairs at Amirkabir University. He started his Master degree in
Industrial Management at Amirkabir university when he passed the National exam in 1991 and completed this degree in 1992. In late 1992 his scholarship was approved by the ministry of Science to go to UK and continue his PHD in management science and productivity. He started his PHD in 1993 and completed in Manchester Business School in 1993. He moved to Amirkabir university in 1993. Upon his return, he was invited to work appointed as the director of Budget and planning department in the Ministry of Science, Research and Technology. In 2002 he was appointed as the scientific representative and director of Iranian students in the UK and Ireland and continued in this position until October 2007. Over this period he managed many new projects such as Iranian Students Information System (ISIS) and scholarship increase project. In addition he facilitated many academic links between both Iranian and the UK Universities and organise the visit of many academic staff of both countries. He also visited most of UK and Ireland universities to talk with supervisors, head of departments and other officials to explain the Iranian universities academic potentials and facilitate the link at individual, departmental and university levels. He managed to build of a two plus two scheme with Amirkabir and Birmingham universities. In addition, during his tenure in the UK he held several conferences at UK universities. Upon his return from the UK in 2007, he was appointed as the head of Amirkabir College of Management and Technology and continued at this and was held this position until 2012 when the number of students at the institute reached 2,000.He was active in several other responsibilities, including the Executive Manager of National Research Project at Amirkabir University of Technology, Executive Advisor of Technical and Vocational University, Vice Chancellor and Financial, Administrative, and Resource Deputy of the Technical and Vocational University and Chancellor of Technical and Vocational University, from March 2012 to December 2016. He has also acted as the Director of Industrial Engineering and Productivity Research Centre at Amirkabir University of Technology, from June 2012 which continues. In the period that he been a faculty member, he has published 50 journal and 38 conference papers. He has held workshops in the field of management, productivity, administrative structure reform, research methods, organizations structure and human resource management in many private and public organizations.

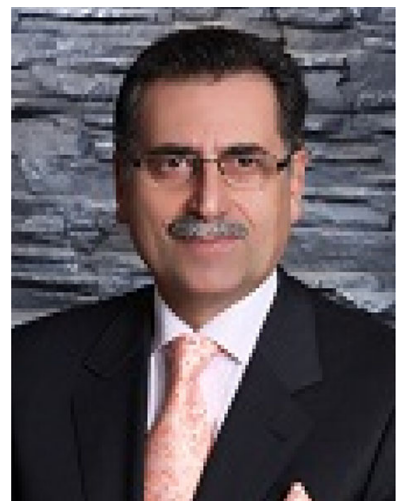

Dr. Kaveh-Mohammad Cyrus is an emeritus assistant professor from department of Industrial Engineering of AmirkabirKabir university.He is referenceable teacher in management, and optimization in Iran. He has published more than 12 books, more than 12 ISI papers, more than 53 international conference papers up to know.He has held many courses in the field of management, enterprise engineering, optimization, productivity, and Strategic Management and Planning in many private and public organizations.Also, he has published many original and review papers in Iranian journals during last 32 years ago up to now. 